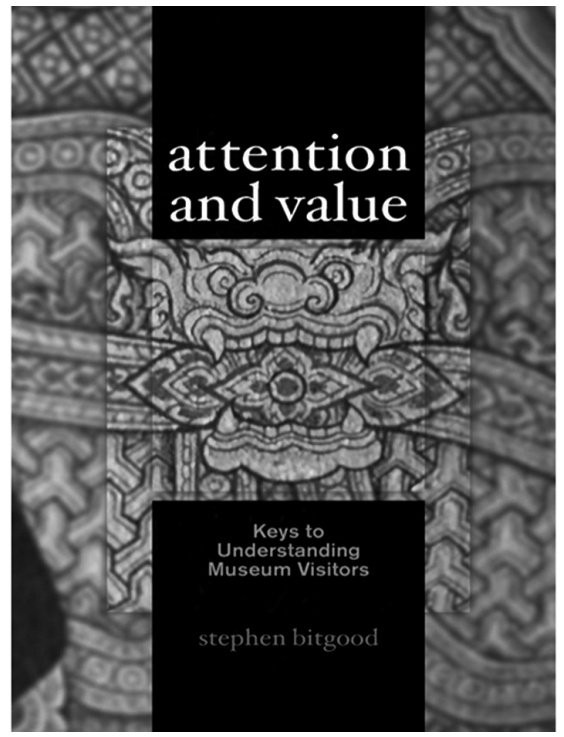

FIGURA 1. Portada de Attention and Value: Keys to Understanding Museum Visitors, Stephen Bitgood, Walnut Creek, Left Coast Press, 2013.

\section{Attention and Value:} Keys to Understanding Museum Visitors (Atención y valor: claves para comprender a los visitantes de museos), de Stephen Bitgood

Attention and Value: Keys to Understanding Museum Visitors, by Stephen Bitgood

\section{Manuel Gándara Vázquez}

Escuela Nacional de Conservación, Restauración y Museografía (ENCRyM), Instituto Nacional de Antropología e Historia (INAH), México gandarav@prodigy.net.mx

\section{Resumen}

Se comenta brevemente Attention and Value: Keys to Understanding Museum Visitors (Atención y valor: claves para comprender a los visitantes de museos), el libro más reciente de uno de los pioneros y líderes en el campo de estudios de visitantes en museos, Stephen Bitgood, que en esta obra reúne, condensa y articula en un argumento unificado ideas que ya había expuesto y que estaban dispersas en numerosos artículos. El volumen describe con detalle un modelo, Ilamado de atención-valor, que intenta explicar por qué los visitantes ponen atención a ciertos elementos de una exposición y no a otros. La importancia de este modelo es que postula lo siguiente: si en la experiencia de poner atención a lo que se le presenta el visitante no la fija durante tiempo suficiente, es casi imposible que aprenda algo de ella. Así, se contiende que si bien la atención no garantiza el aprendizaje, sí es su precondición sine qua non.

\section{Palabras clave}

estudios de visitantes; modelo atención-valor; aprendizaje en museos; museología

\section{Abstract}

This is brief comment on Attention and value: keys to understanding museum visitors, the latest book of Stephen Bitgood, one of the pioneers and leaders in the field of visitor studies. In this volume he brings together and condenses ideas previously presented and dispersed in numerous publications, which are articulated into a unified argument. He exposes his "attention-value" model in detail, in order to explain why visitors devote their attention to some elements of an exhibition rather to others. This model is important since if the visitor does not pay attention enough, then it will be difficult to learn something from that experience. Its contention is therefore that while attention does not guarantee learning, it is its precondition.

\section{Key words}

visitor studies; attention-value model; learning in museums; museum studies 
S tephen Bitgood es, indudablemente, uno de los pioneros de los modernos estudios de públicos: se cuenta entre los fundadores de la Visitor Studies Association (VSA, Asociación de Estudios de Visitantes), de la revista Visitor Studies y el congreso más importante sobre esta materia (Visitor Studies Conference). ${ }^{1}$ La obra del hoy profesor emérito en psicología en la Jackson State University (ISU, Universidad Estatal de Jackson, Estados Unidos de América) (JSU 2015) se remonta a más de tres décadas, a lo largo de las cuales ha explorado temas antes desatendidos en la museología (v. gr. Bitgood 1988; 2011). Un ejemplo de ello es su temprana insistencia sobre la importancia de la orientación cognitiva y espacial, para lo que ha incorporado en los museos hallazgos de la disciplina del wayfinding ${ }^{2}$ (Bitgood 1988). Aunque originalmente a su trabajo se le podría considerar más cercano a la psicología conductual, Bitgood (2011) ha transitado a lo que en el estudio de la interacción entre el entorno y la conducta en grupo - en este caso, el museo y sus visitantes- hoy denomina el diseño social. El eje sobre el que gira su obra es, sin embargo, cómo mejorar el aprendizaje en el museo, con especial interés en el papel que juega la atención en este proceso. Ése es el centro del libro que hoy reseño Attention and Value: Keys to Understanding Museum Visitors (Atención y valor: claves para comprender a los visitantes de museos) (Bitgood 2013), que tras retomar elementos previamente publicados y dispersos en la literatura, se articulan en un argumento coherente en torno del modelo de su autor, de atención-valor.

\footnotetext{
${ }^{1}$ La conferencia se enfoca en un aspecto diferente de los estudios de visitantes y tiene una sede distinta cada año. En el 2016 tendrá lugar en Boston y se dedicará a la revolución de los datos (VSA 2015).

${ }^{2}$ Literalmente, encontrando el camino: estudia la orientación espacial; también se la conoce como diseño ambiental o diseño en apoyo a la orientación espacial, y en ocasiones, mal traducida en español, como señalética.
}

El libro está organizado en cuatro partes y un total de dieciséis capítulos. En la primera de aquéllas (Bitgood 2013:9-75), aborda lo que sabemos sobre la atención de los visitantes en el museo, para lo que Bitgood rastrea tanto la historia de los estudios al respecto como las teorías y los modelos que se han propuesto para determinar su efecto sobre el aprendizaje. La sección concluye con una síntesis del modelo que el autor ha venido desarrollando, Ilamado de atención-valor (Bitgood 2013:64), que, en síntesis, se trata de un modelo que intenta ser incluso predictivo de conductas como la lectura de textos (cédulas) en los museos, asunto que se discute en la segunda sección (Bitgood 2013:77-108). Bitgood (cfr. 2010) ya había dado a conocer avances sobre la idea fundamental del libro: si la exhibición no logra atraer y mantener la atención del público, será prácticamente imposible que apoye el aprendizaje. Aunque poner atención no garantiza que haya aprendizaje, éste es imposible sin atender. A tal mecanismo subyace, no obstante, una relación costo/beneficio: si al focalizar la atención no se encuentra una recompensa suficiente, el visitante se "desengancha" y el aprendizaje no ocurrirá (a esta idea regresaré más adelante). La tercera sección (Bitgood 2013:110-152) aprovecha el modelo para analizar maneras de promover que los visitantes se mantengan atentos, y la cuarta (Bitgood 2013:154-285), en tanto examina las razones por las que se pierde la atención, cierra con sugerencias sobre cómo a través de una adecuada museografía es posible alentar y retener la atención de los públicos. El libro contiene dos apéndices: el primero (Bitgood 2013:187), con ejemplos de textos usados en ciertos experimentos descritos a lo largo de sus páginas; el segundo (Bitgood 2013:189) está conformado por una lista cotejable para diagnosticar si una exposición realmente logra mantener la atención de sus visitantes, esto es, por un recuento, de gran utilidad, de recomendaciones que se han de seguir.
El modelo atención-valor propone ambas variables como claves para las diferentes metas que, se ha sugerido, tiene el museo (Bitgood 2013:6465): 1) proporcionar experiencias de aprendizaje significativas; 2) cambiar actitudes; 3) crear experiencias intelectuales y anímicas que estimulen la curiosidad y la emoción, y 4) facilitar la inmersión, el estado de flujo, una sensación de renovación, etc. (es decir, variables relacionadas, más bien, con la contemplación). Independientemente de cuál o cuáles de ellas se elijan, argumenta Bitgood (2013:65), todas tienen como prerrequisito la atención. Fiel a sus raíces empiristas, ${ }^{3}$ arguye que mientras que solamente podemos inferir los resultados de la atención (como el aprendizaje, que no con facilidad es directamente observable en el museo), sí contamos con medidas de la atención que el público presta a lo exhibido.

La atención ocurre en tres etapas o estadios —en inglés, stages - (Bitgood 2013:12): captura, foco y lo que sería la traducción literal de engagement, que coloquialmente entendemos como enganchamiento: compromiso. Como ha mostrado, entre otras, la psicología cognitiva $(v$. gr. Ausubel 1968) y su aplicación en el campo de la interpretación en museos (Ham 1999), la atención es un fenómeno selectivo (queda implícito que la mente, al enfocarse en algo, desatiende lo demás). A mí me gusta hacer la analogía con una lámpara de mano en una habitación a oscuras: o se apunta a un lado o se apunta a otro. Imposible iluminar todo simultáneamente. $\mathrm{Si}$, en cambio, se la mueve de manera desordenada de una parte a otra, el resultado será

\footnotetext{
${ }^{3}$ Utilizo el término de forma descriptiva, no peyorativa, para referirme a una postura epistemológica en la que se asume que hay una "observación directa", a diferencia de una en que no hay observaciones "neutrales". Para una postura de este tipo, el aprendizaje no se podría observar directamente, sino sería un "efecto inferido" de la atención, que sí es "observable" y mesurable directamente.
} 
una mente "distraída", como decía el pragmatista William James, citado por Bitgood (2013:12).

De ahí las dos ideas centrales detrás del modelo: el éxito de una exhibición depende de su acierto al manejar la atención de sus visitantes, y la motivación primaria (aunque no la única) para poner atención es el valor percibido de hacerlo, que se traduce en la fórmula: utilidad (o beneficio) dividido entre costo (Bitgood 2013:12). La evidencia de años de investigación lleva a Bitgood al siguiente enunciado: "los visitantes parecen hacer ese cálculo, a veces de manera inconsciente, antes de decidir atender algo en la exposición" (Bitgood 2013:60). Para apoyarlo, refiere diferentes teorías en distintos campos que llegan a conclusiones similares: la relación costo/beneficio está en la base de la decisión de atender (manteniendo, por supuesto, otros factores externos constantes y condiciones adecuadas: aunque se quiera atender, será difícil hacerlo si existen distractores ambientales). De hecho, varias de las propuestas del final del libro tratan precisamente sobre cómo evitar que una museografía ${ }^{4}$ inadecuada actúe en contra de la atención (Bitgood 2013:176-214).

Bitgood (2013:17) define la atención como:

Un grupo de procesos psicológicos: 1) que involucran un continuo de tres etapas (captura, foco y compromiso); 2) en que cada estadio es sensible a una combinación única de factores; 3) en el que cada uno de esos factores está motivado por una interacción por otros más relacionados con la persona (sus valores personales, intereses, experiencias pasadas, $y$ sus procesos perceptual, cognitivo, afectivo y de toma de decisiones), así como del entorno (influencias sociales, diseño museográfico y arquitectónico); y 4) que se miden por

${ }^{4}$ En la tradición anglosajona no se habla de museografía, sino de museum design. He traducido liberalmente el término, para conectarlo con el que usamos en Iberoamérica. diferentes indicadores o variables dependientes (aproximarse a un objeto, detenerse, tiempo destinado a ver, leer, platicar con otros, pensar; por pruebas de aprendizaje y memoria; por escalas de rangos, etc.) que tienen diferentes respuestas (indicadores) en cada estadio.

El autor elabora cada uno de estos elementos definitorios en diferentes partes del libro; se reseña la investigación detrás de cada uno y los hallazgos principales, que finalmente se integran en el modelo atenciónvalor (Bitgood 2013:64-73), el cual se expresa como una sencilla fórmula: atención = detección + valor, que se lee como las probabilidades de poner atención a un elemento están directamente relacionadas con: 1) la facilidad de detección de ese elemento, y 2) el valor relativo asociado con la experiencia de comprometer la atención en ese elemento, comparado con diferentes alternativas posibles. Esa ponderación varía en cada una de las tres etapas del proceso: la detección, en el de captura, y el valor, en el de compromiso (Bitgood 2013:64-73).

La atención pasa de estar desenfocada o dispersa a un estado en que se enfoca y el proceso de información se profundiza: dos de los prerrequisitos del aprendizaje; para ello, y poder evaluar si vale la pena atender el elemento -lo que depende de procesos sensorial-perceptuales que hay que favorecer y no obstaculizar-, éste primero debe detectarse (Bitgood 2013:66). Un aspecto crítico, entonces, estriba en la forma en que los visitantes hacen una búsqueda visual en el entorno. Pero el elemento clave será el valor percibido en atender, que varía de persona a persona, dado que lo determinan el componente personal y el del propio entorno social y físico, lo que, de acuerdo con Bitgood (2013:66), implica un cálculo entre el valor de hacerlo contra el tiempo y el esfuerzo requeridos.

Cada una de estas etapas y sus variables asociadas se analizan en la obra, como también se explica la manera de medirlas y cómo el diseño de la exposición puede actuar a favor o en contra del proceso. Se examinan algunos factores que si bien normalmente no se estudian — como la facilidad de orientarse o circular en la exposición-, impactan sobre el costo de poner atención y, con ello, afectan el valor de hacerlo (Bitgood 2013:83-93). El modelo, dice el autor, tiene así aplicaciones tanto diagnósticas como analíticas, e incluso, hasta cierto punto, predictivas (Bitgood 2013:94-154), y apunta a variables que no están completamente entendidas, como la de los patrones de barrido visual ${ }^{5}$ o el efecto de la fatiga, o de saciación ${ }^{6}$ —en inglés, satiation-, o saturación, que degradan la posibilidad de mantener la atención (Bitgood 2013:155-165).

Éste es un libro que acaso no resultará igualmente atractivo a todos los lectores. En particular aquellos que prefieren algo más cercano al lado hermenéutico en las ciencias sociales, encontrarán que Bitgood no oculta su pretensión de cientificidad "pura y dura", lo que posiblemente choque con sus sensibilidades metodológicas. Pero los argumentos presentados están bien fundamentados y son fuertes, lo cual ameritará, antes de descartarlo como "positivista", argumentos en contra que al menos sean tan fuertes y, agregaría yo, útiles, como los que el autor presenta.

Attention and Value es una excelente fuente de reflexiones sobre cómo los visitantes deciden o no poner atención a lo que les proponemos los museólogos, y se une a una creciente literatura que nos lleva a reconsiderar la arrogancia de pensar que lo que diseñamos y montamos en nuestras exposiciones determina por sí solo la experiencia del visitante, como han argumentado con vigor Falk y Dierking (1992; 2013), entre otros.

\footnotetext{
${ }^{5}$ Se le llama barrido visual a la trayectoria que sigue el ojo al explorar el campo visual, la cual es factible rastrear y trazar hoy en día con detectores de movimiento de la retina.

${ }^{6}$ El término no ha sido aceptado en el Diccionario de la Real Academia.
} 
En tanto implica un modelo de costo/beneficio, el de Bitgood se beneficiará (o perjudicará) de una lógica que recuerda mucho la de la economía capitalista, en donde se busca maximizar siempre el valor (quizá algunos lectores encontrarán ofensivo aplicar este modelo a un contexto semisagrado, como ciertos sectores de la profesión siguen estimando los museos y los sitios patrimoniales). Habrá que leer su libro, sin embargo, porque, si se me permite, aporta un gran valor hacerlo, aunque sea sólo para debatir las ideas que presenta.

\section{Referencias}

Ausubel, David P.

1968 Educational Psychology: A Cognitive View, Nueva York y Toronto, Holt, Rinehart \& Winston.
Bitgood, Stephen

1988 "Problems in visitor orientation and circulation", en Stephen Bitgood, James T. Roper y Arlene Benefield (eds.), Visitor Studies-1988: Theory, Research, and Practice, Jacksonville, Center for Social Design, 155-170.

2010 "An analysis of visitor circulation: movement patterns and the General Value Principle", Curator, 49 (4):463-475.

2011 Social Design in Museums: The Psychology of Visitor Studies, Edimburgo, MuseumsEtc.

2013 Attention and Value: Keys to Understanding Museum Visitors, Walnut Creek, Left Coast Press.

Falk, John H. y Lynn D. Dierking 1992 The Museum Experience, Washington, Whalesback Books. 2013 The Museum Experience Revisited, Walnut Creek, Left Coast Press.
Ham, Sam

1999 "Cognitive Psychology and interpretation: synthesis and application", en Eilean Hooper-Greenhill, The Educational Role of the Museum, Nueva York, Routledge, 107117.

JSU

2015 "Steve Bitgood", Jacksonville State University [página web], documento electrónico disponible en [www.jsu.edu/psychology/bitgood. html], consultado el 5 de mayo del 2015.

VSA

2015 "The data revolution", Visitors Studies [página web], documento electrónico disponible en [http:// www.visitorstudies.org/conference], consultado en diciembre del 2015.

\section{Síntesis curricular del/os autor/es}

\section{Manuel Gándara Vázquez}

Escuela Nacional de Conservación, Restauración y Museografía (ENCRyM), Instituto Nacional de Antropología e Historia (INAH), México gandarav@prodigy.net.mx

Doctor en diseño y nuevas tecnologías (Universidad Autónoma Metropolitana-Azcapotzalco [UAM-A], México), así como en antropología (Escuela Nacional de Antropología e Historia [ENAH], Instituto Nacional de Antropología e Historia [INAH], México). Ha investigado sobre problemas teóricos y epistemológicos de la arqueología y, más recientemente, acerca de la divulgación como herramienta de conservación del patrimonio arqueológico, en particular con nuevos enfoques de comunicación y tecnologías. Es pionero, desde 1974, en cómputo educativo. Ha desarrollado numerosos programas multimedia para museos, así como software educativo comercial. Ha publicado también sobre la interacción humano-computadora, el diseño de interfases y la usabilidad. Dirigió la ENAH-INAH y el Centro de Tecnología y Medios Educativos (CTME-SEP), y coordinó el Centro de Cultura Digital de IntTelmex (los tres, en México). En la actualidad labora en el posgrado en museología de la ENCRYM-INAH.

Postulado/Submitted 21.05.15

Aceptado/Accepted 04.01.16

Publicado/Published 31.03.2016

s

Intervención, Revista Internacional de Conservación, Restauración y Museología, año 7, número 13, enero-junio de 2016, se terminó de publicar electrónicamente el 31 de marzo de 2016, Ciudad de México. 